\title{
Toracoscopia aplicada à ressecção de fragmento pulmonar com o auxílio de sutura mecânica em eqüinos
}

\author{
[Thoracoscopy for resection of lung fragment in horses with the aid of mechanical suturing] \\ A.L.V. Zoppa ${ }^{1}$, J. Alvarenga ${ }^{1}$, R.S.F. Cruz ${ }^{2}$, T.S.L. Machado ${ }^{3}$, L.C.L.C. Silva ${ }^{1}$ \\ ${ }^{1}$ Faculdade de Medicina Veterinária e Zootecnia - USP \\ Av. Prof. Orlando Marquês de Paiva, 87 - Butantã \\ 05508-000 - São Paulo, SP \\ ${ }^{2}$ Médico veterinário do Hospital Veterinário - FMVZ-USP- São Paulo, SP \\ ${ }^{3}$ Aluna de pós-graduação - FMVZ-USP-São Paulo, SP
}

\begin{abstract}
RESUMO
Este trabalho visou à ressecção de fragmento dorsocaudal do lobo pulmonar caudal esquerdo com o auxílio de sutura mecânica por via toracoscópica. Foram utilizados 10 eqüinos clinicamente sadios, os quais foram sedados e mantidos em estação. $\mathrm{O}$ procedimento foi realizado no hemitórax esquerdo, por três acessos previamente programados: o primeiro acesso foi no $12^{\circ}$ espaço intercostal (EIC), o segundo no $14^{\circ}, 15^{\circ}$ ou $16^{\circ}$ EIC e o terceiro no $14^{\circ}$ ou $15^{\circ}$ EIC, conforme a necessidade de posicionamento dos instrumentos. Em todos os animais foi possível controlar o pneumotórax, sendo observada evolução clínica satisfatória durante o período pós-operatório, exceto em um animal. Os resultados obtidos indicam que, por meio da videotoracoscopia, pode-se realizar ressecção pulmonar parcial com uso de sutura mecânica em eqüinos e seu emprego na rotina hospitalar poderá contribuir para melhor compreensão e controle de enfermidades sediadas na cavidade torácica.
\end{abstract}

Palavras-chave: eqüino, cirurgia torácica, toracoscopia, pulmão, lobectomia

\begin{abstract}
In this study, a dorsocaudal portion resection of the left caudal lung lobe was performed using an endoscopic stapler by thoracoscopic procedure. Ten healthy horses, eight males and two females were evaluated by clinical examination and submitted to the surgical procedure. The animals were sedated and maintained in standing position. The procedure was done in the left hemitorax using three access previously studied: the first portal was done at the 12th intercostal space (IS); the second one at the 14th, 15th, or 16th IS; and the third one at the 14th or 15th IS, in accordance with the necessity of positioning the instruments. The employed surgical technique seemed to be adequate in all animals, since it helped controlling the existing pneumothorax; furthermore, clinical recovery during the postoperative period was satisfactory, except for one animal. These results suggest that thoracoscopic may successfully be employed for partial lung resection in horses, using endoscopic staplers, and its practical use may contribute to the knowledge and resolution of thoracic diseases.
\end{abstract}

Keywords: equine, thoracic surgery, thoracoscopy, lung, lobectomy

\section{INTRODUÇÃO}

As primeiras toracoscopias, descritas por Jacobaeus, datam de 1910, tiveram o objetivo de realizar adesiólises no tratamento de pacientes com tuberculose pulmonar (Dujmusic, 1950). Na espécie eqüina, os primeiros relatos datam da década de 80, realizados por Mansmann e Bernard-Strother (1985) e Mackey e Wheat (1985).

Recebido em 24 de maio de 2007

Aceito em 4 de abril de 2008

E-mail: alzoppa@usp.br 
Dentre as indicações da toracoscopia em eqüinos, citam-se: avaliação em casos de pleuropneumonias (Mansmann e BernardStrother, 1985; Byars e Becht, 1991), de neoplasias intratorácicas, de aderências, em metástases tumorais e em pericardites (Mackey e Wheat, 1985; Potter e Hendrickson, 1999), para biópsias pulmonares e pleurais (Hendrickson e Wilson, 1996; Potter e Hendrickson, 1999), pleuris bacteriana (Potter e Hendrickson, 1999), na drenagem de abscessos (Hendrickson e Wilson, 1996; Potter e Hendrickson, 1999), para a avaliação de corpos estranhos e fístulas broncopleurais (Byars e Becht, 1991), assim como na colocação e avaliação de drenos torácicos (Vachon e Fischer, 1998; Baccarin et al., 2003).

As indicações de biópsia pulmonar pela ressecção de fragmento cuneiforme em eqüinos incluem: lacerações pulmonares iatrogênicas ou traumáticas, nódulos pulmonares e vesículas na superfície pulmonar, grandes para ressecção com técnica de ligadura do tipo Endoloop (Potter e Hendrickson, 1999).

Demmy et al. (1997), após a realização de 50 toracoscopias em pacientes humanos, afirmaram que a biópsia pulmonar por toracoscopia é mais precisa quando comparada à biópsia aspirativa com agulha, e mais produtiva que a por broncoscopia.

A toracoscopia pode ser realizada com o animal em estação, sob efeito de anestesia local associada a sedativos e analgésicos (Mackey e Wheat, 1985; Mansmann e Bernard-Strother, 1985; Freeman, 1991). A dose recomendada de xilazina associada a butorfanol é de respectivamente, $0,55-0,85 \mathrm{mg} / \mathrm{kg}$ e $0,01 \mathrm{mg} / \mathrm{kg}$, ambos por via intravenosa (IV) (Freeman, 1991). Zoppa et al. (2001) utilizaram, como protocolo para toracoscopia, romifidina na dose de $0,12 \mathrm{mg} / \mathrm{kg}$, por via IV, precedida de analgesia com meperidina na dose de $1,0 \mathrm{mg} / \mathrm{kg}$, por via intramuscular (IM)

Segundo Mackey e Wheat (1985), na realização da toracoscopia em eqüinos, o acesso para introdução da óptica é realizado através de incisão de aproximadamente $2 \mathrm{~cm}$. Em seguida, punciona-se a pleura costal com pinça hemostática e introduz-se uma cânula mamária no interior da cavidade pleural (Mansmann e
Bernard-Strother, 1985). De acordo com Peroni et al. (2001), a indução de pneumotórax insuficiente pode predispor lesão do parênquima pulmonar quando da introdução do trocarte.

Lugo et al. (2002) adotaram a técnica de triangulação em 10 eqüinos submetidos à toracoscopia para ressecção pulmonar, sendo que o primeiro acesso foi no $13^{\circ}$ espaço intercostal e os outros dois feitos de modo que se mantivesse a distância de dois ou três espaços intercostais entre eles para não restringir a manipulação dos instrumentos.

A utilização de cuba com solução fisiológica aquecida, para prevenir que a lente da óptica embace, causada pela diferença de temperatura entre a cavidade intratorácica e o ambiente, foi recomendada por Landreneau et al. (1992).

A necessidade de angulação do conjunto trocarte/óptica causa desconforto aos animais pela compressão dos nervos intercostais. Este fato foi relatado por Peroni et al. (2001), que ao retornarem o trocarte à posição de $90^{\circ}$ em relação ao tórax, observaram cessação do estímulo doloroso. Lugo et al. (2002) minimizaram esse desconforto com a administração de anestésico local na região da incisão.

Para efetuar a ressecção de fragmento pulmonar, Lugo et al. (2002) utilizaram instrumento de sutura mecânica de $45 \mathrm{~mm}$ de comprimento e grampos de $4,1 \mathrm{~mm}$, sendo necessários dois disparos para destacar o fragmento pulmonar. De acordo com a necessidade, a posição da pinça atraumática e do instrumento de sutura mecânica foi trocada para facilitar a manipulação do pulmão (Lugo et al., 2002).

As complicações relatadas no transoperatório foram a laceração iatrogênica do diafragma causada pela passagem do trocarte, levando a hemotórax e pneumotórax residual (Lugo et al., 2002), a punção acidental da artéria intercostal e o mau posicionamento dos trocartes (Faunt et al., 1998). Peroni et al. (2001) não observaram casos de pneumotórax bilateral no transoperatório em nenhum dos eqüinos submetidos à toracoscopia.

Após a retirada do endoscópio, o ar presente no interior do tórax pode ser removido por aspirador, evitando-se, assim, a ocorrência de 
pneumotórax residual. A aspiração pode ser acompanhada visualmente antes da retirada do endoscópio (Mansmann e Bernard-Strother, 1985; Peroni et al., 2001). Faunt et al. (1998) e Peroni et al. (2001) citaram a formação de enfisema subcutâneo como complicação presente durante o período pós-operatório.

Visando a reavaliar o local da ressecção pulmonar, Lugo et al. (2002) realizaram toracoscopia no $14^{\circ}$ dia de pós-operatório, sendo observada presença de fibrose na pleura pulmonar no local da ressecção.

Segundo Lugo et al. (2002), a desvantagem relacionada ao procedimento de ressecção pulmonar com sutura mecânica, por via toracoscópica, é a possibilidade de realizar o procedimento apenas na periferia do órgão.

Este trabalho visou à realização de ressecção do fragmento dorsocaudal do lobo pulmonar caudal esquerdo com auxílio de sutura mecânica por via toracoscópica, para avaliar a possibilidade de indução gradual do pneumotórax, a viabilidade da técnica cirúrgica em eqüinos, mantidos sedados e em estação, a avaliação da presença de pneumotórax residual, e a utilização de material de sutura mecânica usado em procedimentos com a espécie humana.

\section{MATERIAL E MÉTODOS}

Foram utilizados 10 eqüinos adultos, oito machos e duas fêmeas, com pesos entre 322 e $492 \mathrm{~kg} \quad(428,70 \pm 51,36)$, idades variáveis e clinicamente sadios ${ }^{1}$.

Os eqüinos passaram por exame físico préoperatório quando foram registradas: freqüências cardíaca e respiratória, temperatura corpórea, coloração de mucosas e tempo de preenchimento capilar. Colheu-se sangue para realização do hemograma e fibrinogênio plasmático, seguido de exame ultra-sonográfico do tórax.

O protocolo anestésico usado, após jejum alimentar e hídrico de $12 \mathrm{~h}$, consistiu em sedação com romifidina ${ }^{2}$ na dose de 0,06 a $0,1 \mathrm{mg} / \mathrm{kg}$,

\footnotetext{
${ }^{1}$ Aprovado pela Comissão de Bioética da FMVZ-USP em 28/05/2003 (Protocolo 297/2003).

${ }^{2}$ Sedivet ${ }^{\circledR}$ - Boehringer Ingelhein.
}

analgesia com tartarato de butorfanol ${ }^{3}$ na dose de $0,05 \mathrm{mg} / \mathrm{kg}$, administrados por via intravenosa (IV). Realizou-se anestesia local infiltrativa com $10 \mathrm{ml}$ de lidocaína ${ }^{4}$ a $2 \%$, em cada local de acesso escolhido. Os animais foram mantidos em tronco de contenção durante o procedimento.

Após tricotomia da região do gradil torácico esquerdo, realizou-se antissepsia com iodopovidina ${ }^{5}$ e álcool. Abordou-se inicialmente o $12^{\circ}$ espaço intercostal (EIC), para realização do primeiro acesso. Após incisão de $3 \mathrm{~cm}$ em pele e fáscia muscular, utilizou-se técnica de introdução assistida da cânula, por meio de trocarte modelo Endo $\operatorname{Tip}^{\circledR 6}$, que promove divulsão romba dos planos musculares, fáscia endotorácica e pleura costal por meio de movimentos de rotação. Realizada a introdução da óptica ${ }^{7}$ e inspecionada a cavidade torácica, foram criados dois novos acessos para possibilitar a preensão e o grampeamento do segmento pulmonar. Os locais exatos para incisão na pele e passagem dos outros dois trocartes ${ }^{8}$ foram selecionados durante o procedimento.

Determinados os três acessos, a óptica, a pinça de preensão tipo Babcock ${ }^{9}$ e o grampeador endoscópico ${ }^{10}$ foram posicionados para execução da técnica, quando se observou a necessidade de posicionamento da óptica pelo acesso mais caudal (Fig. 1). Posicionados os instrumentos, a equipe cirúrgica foi disposta, de modo que o cirurgião responsabilizou-se pela manipulação da pinça de preensão e o grampeador, enquanto o auxiliar foi o responsável pela manutenção do enquadramento e da qualidade da imagem.

Uma vez pinçado o lobo caudal esquerdo na sua face dorsocaudal, este foi apresentado para posicionamento do grampeador endoscópico. Após o primeiro disparo, avaliou-se a região seccionada do parênquima pulmonar quanto à

${ }^{3}$ Torbugesic $^{\circledR}-$ Fort Dodge.

${ }^{4}$ Cloridrato de Lidocaína 2\% - Medicamento Genérico Hipolabor.

${ }^{5}$ Riodeine ${ }^{\circledR}$ - Rioquímica Indústri Farmacêutica.

${ }^{6}$ Cânula de $10,5 \mathrm{~cm}$ X e $10 \mathrm{~mm}$, Endo TIP ${ }^{\circledR}$, Karl Störz.

${ }^{7}$ Óptica rígida de $30 \mathrm{~cm} \times 10 \mathrm{~mm}$ e $0^{\circ}$ de ângulo de visão, Asap.

${ }^{8}$ Trocarte de $10 \mathrm{~cm}$ X $11 \mathrm{~mm}$, Endopath 511, Ethicon EndoSurgery.

${ }^{9}$ Pinça tipo Babcock, de $33 \mathrm{~cm}$ X $10 \mathrm{~mm}$, com cremalheira, Wisap.

${ }^{10}$ Endo Grampeador Linear Cortante, Endopath EZ 45, Ethicon Endo-Surgery. 
ocorrência de sangramento. O grampeador foi retirado e recarregado, realizando-se o segundo disparo e nova avaliação da linha de sutura. Novamente, avaliaram-se o sangramento e o posicionamento dos grampos. $\mathrm{O}$ fragmento pulmonar ressecado foi retirado através do segundo acesso onde estava o grampeador, utilizando-se pinça tipo $\mathrm{Claw}^{11}$.

Após a retirada do fragmento pulmonar, removeu-se o ar intratorácico com auxílio de um aspirador cirúrgico, para minimizar o pneumotórax residual. Após a retirada da cânula e do endoscópio, a musculatura foi suturada em padrão de Sultan, utilizando-se fio monofilamentar de náilon $2.0^{12}$, e as bordas da pele aposicionadas em padrão interrompido simples, utilizando-se o mesmo fio.

O tempo de procedimento cirúrgico foi acompanhado nos 10 animais, tomando-se como início o momento da sedação, e como término a finalização da sutura de pele. Os cuidados pósoperatórios incluíram a aplicação de fenilbutazona $^{13}$, na dose de $2,2 \mathrm{mg} / \mathrm{kg}$, IV, a cada $24 \mathrm{~h}$, durante três dias. Fez-se o acompanhamento clínico e realizaram-se curativos diários da ferida cirúrgica até a sua cicatrização. Os pontos cutâneos foram retirados 10 dias após o procedimento cirúrgico.

\section{RESULTADOS E DISCUSSÃO}

Em relação ao protocolo anestésico, houve necessidade de suplementação com romifidina nos animais 3, 6 e 9, e com tartarato de butorfanol nos animais 5, 6 e 8 . Essa variação deveu-se à oscilação do tempo cirúrgico, de 35 a $77 \mathrm{~min}$ (média de 54min), e à necessidade de maior manipulação do instrumental cirúrgico, em alguns animais. Este protocolo propiciou boa sedação e analgesia durante o procedimento, sugerindo a associação de $\alpha_{2}$ - agonistas e derivados opióides, conforme foi relatado por Zoppa et al. (2001), à infiltração de anestésico local.

\footnotetext{
${ }^{11}$ Pinça tipo Claw, de $33 \mathrm{~cm} \times 10 \mathrm{~mm}$, com cremalheira, Wisap.

${ }^{12}$ Mononylon Ethilon 2-0, Ethicon.

${ }^{13}$ Equipalazone $^{\circledR}$ - laboratório Marcolab.
}

O acesso inicial para introdução da cânula e óptica, diferente do relatado por Mackey e Wheat (1985), foi realizado através de incisão da pele e fáscia muscular de, aproximadamente $3 \mathrm{~cm}$, e introdução da cânula Endo Tip ${ }^{\circledR}$, instrumental não utilizado pelos referidos autores. A incisão de pele de $3 \mathrm{~cm}$ deveu-se ao fato de os autores acreditarem que durante episódios de tosse, o ar seria drenado com maior facilidade, prevenindo, assim, a formação de enfisema subcutâneo, fato não confirmado nos animais 1, 2, 7 e 10, e facilitando a passagem do grampeador endoscópico e, posteriormente, a retirada do fragmento pulmonar.

A indução assistida do pneumotórax, com o uso do Endo Tip ${ }^{\circledR}$, tornou o acesso à cavidade pleural seguro, diferente do relatado por Mackey e Wheat (1985), Mansmann e Bernard-Strother (1985) e Peroni et al. (2001), que utilizaram cânula mamária como auxílio para induzir o pneumotórax. A única intercorrência observada foi a laceração superficial de pleura pulmonar no animal 4, no entanto, não ocorreu sangramento ou lesão no parênquima. A facilidade de manipulação aliada à segurança para indução do pneumotórax, permite sugerir que esse instrumental seja parte integrante do equipamento de cirurgia torácica videoassistida.

O pneumotórax foi induzido com ar ambiente e controlado com aspirador acoplado à cânula por equipo de soro, possibilitando visão ampla da cavidade torácica, fato que permitiu a não utilização de insuflador para indução do pneumotórax, como foi sugerido por Faunt et al. (1998). Contrariamente às toracoscopias indicadas com fins diagnósticos, em que a exploração da cavidade torácica necessita de colabamento pulmonar acentuado, as cirurgias toracoscópicas podem ser realizadas com a indução gradual de pneumotórax até viabilizar a realização do procedimento. Dessa forma, evitam-se o desconforto do paciente e episódios de tosse (Peroni et al., 2001).

As cirurgias minimamente invasivas requerem que os acessos sejam escolhidos respeitando a técnica de triangulação, conforme foi sugerido por Lugo et al. (2002), que recomendaram a escolha de um espaço intercostal inicial para inspeção da cavidade torácica e, posteriormente, passagem dos outros trocartes. Nesse sentido, optou-se pelo $12^{\circ}$ espaço intercostal esquerdo, 
que permitiu boa visualização e auxiliou na escolha dos outros acessos e, ainda, possibilitou inspeção das pleuras costal e pulmonar e das estruturas intratorácicas. A imersão prévia da óptica em solução fisiológica aquecida evitou que a lente embaçasse quando dentro da cavidade pleural.

Lugo et al. (2002) ressaltaram, também, a importância da distância de dois a três espaços intercostais entre os trocartes para que a sua manipulação e a visão da cavidade não fossem prejudicadas, o que resultou, neste trabalho, em uma variação na escolha dos outros dois acessos. $\mathrm{O}$ segundo acesso foi direcionado dorsocaudalmente e variou entre o $13^{\circ}$ e $16^{\circ}$ EIC; o terceiro, que variou entre o $14^{\circ}$ e $16^{\circ} \mathrm{EIC}$, foi direcionado ventralmente em relação ao segundo.

De maneira semelhante ao citado por Lugo et al. (2002), foi realizada troca de posicionamento da óptica, passando do primeiro acesso, no $12^{\circ}$ espaço intercostal, para o segundo acesso, dorsocaudalmente.

O pinçamento da borda pulmonar mais caudal com pinça tipo Babcock (Fig. 2), favoreceu o posicionamento do grampeador, sendo que em alguns momentos houve deslizamento da pinça e necessidade de nova preensão. Durante o posicionamento do grampeador, houve necessidade de manipulação sincronizada da pinça de preensão para que não ocorresse a dobra da borda pulmonar colabada. Não foi observada hemorragia no local após o primeiro disparo. Buscou-se posicionamento adequado do grampeador no momento do segundo disparo, de modo que as partes finais da primeira linha de sutura e o início da segunda ficassem sobrepostas, não ocorrendo extravasamento de ar e falha na sutura durante a expansão pulmonar.

A variação no momento do posicionamento do grampeador fez com que, nos animais 1, 2 e 10, fosse necessário um terceiro disparo, devido à observação de manutenção de parte do parênquima pulmonar sem a presença da sutura, que não permitia o isolamento do fragmento. No animal 8, houve a necessidade de um quarto disparo, pelo mesmo motivo citado anteriormente, e no animal 5, a necessidade de intervenção com tesoura de uso endoscópico para liberação do fragmento pulmonar, pois esse se manteve preso pela pleura pulmonar. $\mathrm{O}$ fragmento foi retirado, pelo $12^{\circ}$ espaço intercostal, com auxílio de uma pinça Claw (Fig. 2).

As alterações no posicionamento dos instrumentos foram importantes, pois a permanência dorsocaudal da óptica possibilitou visão suficiente do local que seria ressecado, facilitando a manipulação dos outros instrumentais.

De maneira oposta ao citado por Peroni et al. (2001), foi observado pneumotórax bilateral (Fig. 3) nos animais 2, 4, 5, 7, 9 e 10, sendo necessária aspiração do ar com auxílio de aspirador, durante o procedimento. Nos animais 5, 9 e 10 houve a necessidade de promover uma comunicação dorsocaudal entre os dois hemitórax, com auxílio de tesoura de uso endoscópico, para que a aspiração bilateral do ar fosse mais eficiente. A aspiração por meio de trocarte permitiu acompanhar a expansão do pulmão até a região dorsocaudal, por ocasião da remoção do último trocarte.

Nos animais 1, 2, 7 e 10, foram observadas formações de enfisema subcutâneo durante o procedimento, após apresentarem episódios de tosse, devido à manipulação e movimentação do trocarte e óptica, com o objetivo de explorar a porção cranial da cavidade torácica.

A possibilidade de visibilização direta das estruturas faz os autores acreditarem, assim como Demmy et al. (1997), que a toracoscopia possibilita ressecção precisa da parte pulmonar alterada. Entretanto, os autores concordam com Lugo et al. (2002) no que diz respeito à desvantagem de poder efetuar essa técnica somente na periferia do pulmão, devido às características do grampeador endoscópico e à maneira como ele é posicionado no parênquima pulmonar, o que torna inviável a ressecção em outros locais. 


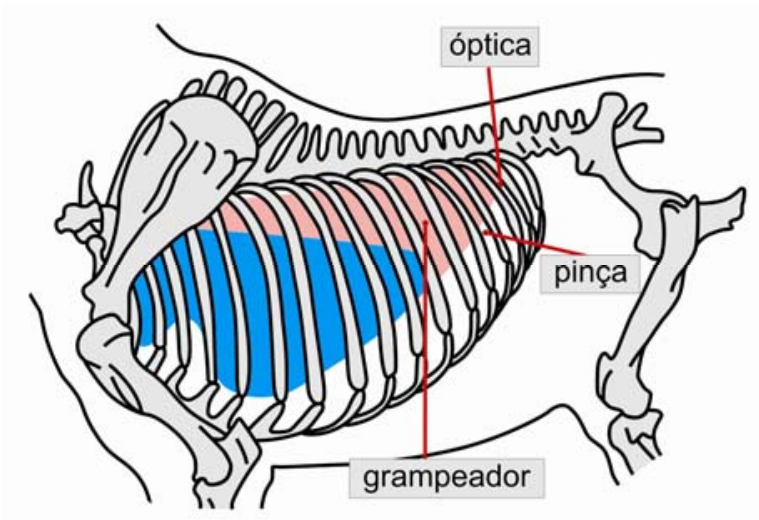

Figura 1. Esquema demonstrando a posição dos instrumentos endoscópicos para realização de ressecção de fragmento pulmonar por toracoscopia em eqüinos. A imagem em rosa representa o pulmão em sua posição anatômica normal, e a azul, o pulmão após a indução do pneumotórax.

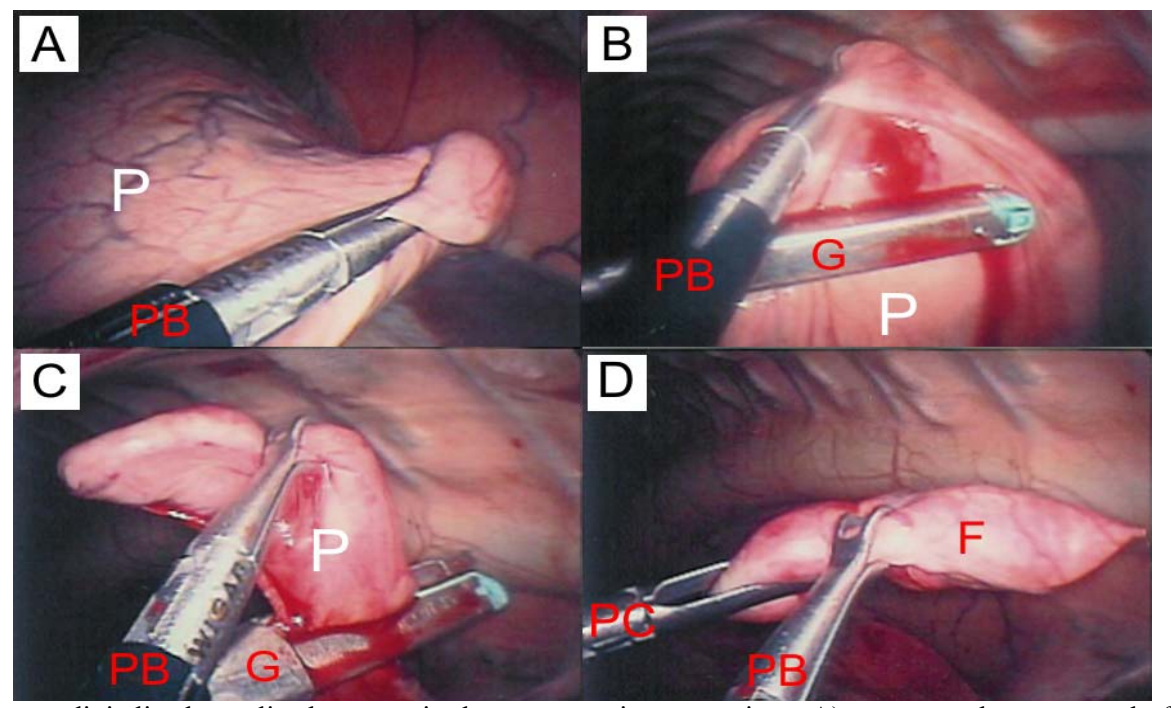

Figura 2. Imagem digitalizada, realizada por meio de toracoscopia, em eqüinos. A) momento de preensão do fragmento do pulmão; B) momento da realização do primeiro disparo com o grampeador endoscópico; C) momento da realização do segundo disparo com o grampeador endoscópico; D) momento da preensão com a pinça Claw.

$\mathrm{P}=$ pulmão; $\mathrm{PB}=$ pinça Babcock; $\mathrm{G}=$ ponta distal do grampeador endoscópio; $\mathrm{PC}=$ pinça $C l a w ; \mathrm{F}=$ fragmento pulmonar.

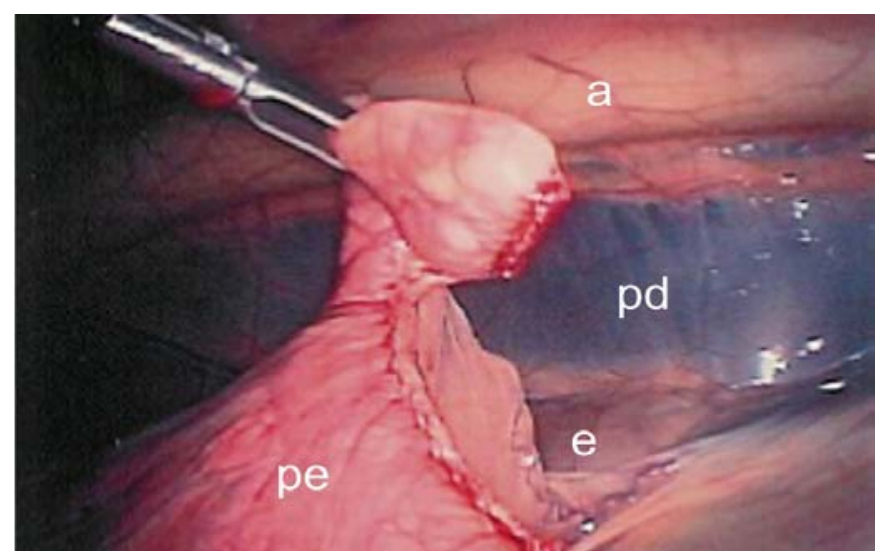

Figura 3. Imagem digitalizada, realizada por meio de toracoscopia em eqüinos, no momento transcirúrgico, após a realização do primeiro disparo com grampeador, com formação de pneumotórax bilateral.

$\mathrm{a}=$ aorta; $\mathrm{pd}=$ pleura mediastínica sem a presença do pulmão direito; $\mathrm{pe}=$ pulmão esquerdo; e= esôfago. 


\section{CONCLUSÕES}

A técnica de ressecção pulmonar em cunha por cirurgia torácica videoassistida é possível de ser realizada em eqüinos mantidos em estação e tronco de contenção. O pneumotórax induzido deve ser suficiente para a realização da técnica cirúrgica escolhida. A ocorrência de pneumotórax bilateral durante o procedimento deve ser prevista e a equipe cirúrgica preparada para uma necessidade de reversão do quadro. As alterações causadas pelo procedimento são momentâneas, seguidas de recuperação rápida, durante o período pós-operatório. A técnica e os instrumentos propostos poderão ser empregados em casos nos quais haja a necessidade de coleta de fragmentos da periferia do parênquima pulmonar e/ou que não permitam a realização de anestesia geral.

\section{REFERÊNCIAS BIBLIOGRÁFICAS}

BACCARIN, R.Y.A.; FERNANDES, W.R.; SILVA, L.C.L.C. et al. Avaliação toracoscópica e ultra-sonográfica da cavidade pleural de eqüinos após colocação de dreno torácico de silicone. Vet. Not., v.9, p.37-44, 2003.

BYARS, T.D.; BECHT J.L. Pleuropneumonia. Vet. Clin. N. Am.: Equine Pract., v.7, p.63-78, 1991.

DEMMY, T.L.; WAGNER-MANN, C.; JAMES, M.A. et al. Feasibility of mathematical models to predict success in video-assisted thoracic surgery lung nodule excision. Am. J. Surg., v.174, p.2023, 1997.

DUJMUSIC, S. Atlas of operative thoracoscopy. Belgrade-Zagreb: Medicinska Knjica, 1950. p.511.

FAUNT, K.K.; JONES, B.D.; TURK, J.R. et al. Evaluation of biopsy specimens obtained during thoracoscopy from lungs of clinically normal dogs. Am. J. Vet. Res., v.59, p.1499-1502, 1998.
FREEMAN, D. E. Pleuroscopy. Vet. Clin. N. Am.: Equine Pract., v.7, p.621-623, 1991.

HENDRICKSON, D. A.; WILSON, D. G. Instrumentation and techniques for laparoscopic and thoracoscopic surgery in the horse. Vet. Clin. N. Am.: Equine Pract., v.12, p.235-251, 1996.

LANDRENEAU, R.J.; MACK, M.J.; HAZELRIGG, S.R. et al. Video-assisted thoracic surgery: basic technical concepts and intercostal approach strategies. Ann. Thor. Surg., n.54, p.800-807, 1992.

LUGO, J.; STICK, J.A.; PERONI, J. et al. Safety and efficacy of a technique for thoracoscopically guided pulmonary wedge resection in horses. Am. J. Vet. Res., v.63, p.1232-1240, 2002.

MACKEY, V.S.; WHEAT, J. D. Endoscopic examination of the equine thorax. Equine Vet. J., v.17, p.140-142, 1985.

MANSMANN, R.A.; BERNARD-STROTHER, S. Pleuroscopy in horses. Mod. Vet. Pract., v.66, p.9-17, 1985.

PERONI, J.F.; HORNER, N.T.; ROBINSON, N.E. et al. Equine thoracoscopy: normal anatomy and surgical technique. Equine Vet. J., v.33, p.231-237, 2001.

POTTER, L.; HENDRICKSON, D.A. Therapeutic video-assisted thoracic surgery. In: FREEMAN, L.J. (Ed). Veterinary endosurgery. St. Louis: Mosby, 1999. cap.9, p.188-191.

VACHON, A.M.; FISCHER, A. Thoracoscopy in the horse: diagnostic and therapeutic indications in 28 cases. Equine Vet. J., v.30, p.467-475, 1998.

ZOPPA, A.L.V.; ALVARENGA, J.; SILVA, L.C.L.C. et al. Toracoscopia em eqüinos: técnica e emprego como método de avaliação da cavidade pleural. Cienc. Rural, v.31, p.825-830, 2001. 